\title{
Facial Phenotype in Children and Young Adults with PHOX2B-Determined Congenital Central Hypoventilation Syndrome: Quantitative Pattern of Dysmorphology
}

\author{
EMILY S. TODD, SETH M. WEINBERG, ELIZABETH M. BERRY-KRAVIS, JEAN M. SILVESTRI, ANNA S. KENNY, \\ CASEY M. RAND, LILI ZHOU, BRION S. MAHER, MARY L. MARAZITA, AND DEBRA E. WEESE-MAYER
}

\begin{abstract}
Department of Pediatrics [E.S.T., E.M.B.-K., J.M.S., A.S.K., C.M.R., L.Z., D.E.W.-M.], Departments of Neurology and Biochemistry [E.M.B.-K.], Rush University Medical Center, Chicago, Illinois 60612; Center for Craniofacial and Dental Genetics [S.M.W., B.S.M., M.L.M.], Department of Human Genetics [M.L.M.], Department of Oral and Maxillofacial Surgery [M.L.M.], University of Pittsburgh, Pittsburgh, Pennsylvania 15219
\end{abstract}

\begin{abstract}
Congenital central hypoventilation syndrome (CCHS) is caused by mutations in $P H O X 2 B$, which is essential for maturation of the neural crest into the autonomic nervous system and is expressed in the dorsal rhombencephalon, a region that gives rise to facial structures. Digital photographs of 45 individuals with PHOX2B-confirmed CCHS, and 45 matched controls were analyzed for 17 linear and 6 angular measurements, and 9 derived indices. Paired $t$ tests were used to compare group means, correlation was calculated between $P H O X 2 B$ polyalanine expansion number and facial measures, and stepwise logistic regression was used to predict case-control and genotype status. CCHS cases differed significantly from controls on 13 variables ( 6 after $p$ value correction: nasolabial angle, upper lip height, lateral lip height, facial index, upper facial index, and presence of inferior inflection of the lateral segment of the upper lip vermillion border). Five variables were able to predict correctly $85.7 \%$ of CCHS cases and $82.2 \%$ of controls: upper lip height, biocular width, upper facial height, nasal tip protrusion, and inferior inflection of the upper lip vermillion border. A negative relationship between number of repeats and four anthropometric measures was observed: mandible breadth, nasolabial angle, lateral lip height, and mandible-face width index. These results suggest a characteristic facial phenotype in children and young adults with CCHS, due to an expansion mutation in PHOX2B. (Pediatr Res 59: 39-45, 2006)
\end{abstract}

$\mathrm{C}^{\mathrm{h}}$ hildren with CCHS typically present in the newborn period "in the absence of primary neuromuscular, lung, or cardiac disease, or an identifiable brainstem lesion," with the characteristic alveolar hypoventilation, altered responses to hypoxemia and hypercarbia, and related evidence of ANS dysfunction/dysregulation (1). A characteristic facies has been anecdotally observed among children with the clinical pheno-

Received April 13, 2005; accepted June 14, 2005.

Correspondence: Debra E. Weese-Mayer, M.D., Professor of Pediatrics at Rush University, Director, Pediatric Respiratory Medicine at Rush Children's Hospital, 1653 West Congress Parkway, Chicago, IL 60612; e-mail: Debra_E_Weese-Mayer@rsh.net

This study was supported by Rush University Dean's Office Summer Research Fellowship and Sigma Xi Outstanding Rush Medical College Student Research Award (E.S.T.), Spastic Paralysis and Allied Diseases of the Central Nervous System Research Foundation of the Illinois-Eastern Iowa District Kiwanis International (E.M.B.-K.), and National Institutes of Health/National Institute of Dental and Craniofacial Research grants R01-DE016148 and P50-DE016215 (M.L.M.).

DOI: 10.1203/01.pdr.0000191814.73340.1d type of CCHS. Qualitatively, the typical CCHS face has been characterized as having a broad, flat, rectangular appearance (Fig. 1). Even among young children, the child with CCHS is identifiable by an upper and mid-face that is short relative to its width, giving the face a characteristic box-like appearance (Figs. 2 and 3). There is also a distinctive pattern to the upper lip with the lateral edges of the vermillion line turning inferiorly. Despite extensive clinical observation, an objective quantitative description of the CCHS facial phenotype is lacking.

Most individuals with CCHS are heterozygous for a polyalanine expansion mutation in the second polyalanine repeat residue of $P H O X 2 B$, with one normal allele having 20 repeats and the affected allele having 25-33 repeats of the polyalanine sequence (2-7). In a small subset of individuals with the CCHS phenotype, unique mutations in $P H O X 2 B$ have been described (2-7). The PHOX2B gene encodes a highly conserved homeobox domain transcription factor (3) with an early embryologic action as a transcriptional activator in promotion of pan-neuronal differentiation and a separate role wherein it represses expression of inhibitors of neurogenesis (8). Because $P H O X 2 B$ is required to express tyrosine hydroxylase, dopamine beta hydroxylase (9), and receptor tyrosine kinase, and to maintain the mammalian aschaete scute homologue gene, $P H O X 2 B$ is considered to regulate the noradrenergic phenotype in vertebrates (10).

CCHS is considered to be a neurocristopathy, or a disease derived from the neural crest. The PHOX2B gene is clearly expressed in the neural crest with an early expression pattern in the rhombencephalon $(10,11)$. Notably, the dorsal rhombencephalon and caudal midbrain give rise to the neural crest that

Abbreviations: ANS, autonomic nervous system; CCHS, congenital central hypoventilation syndrome 


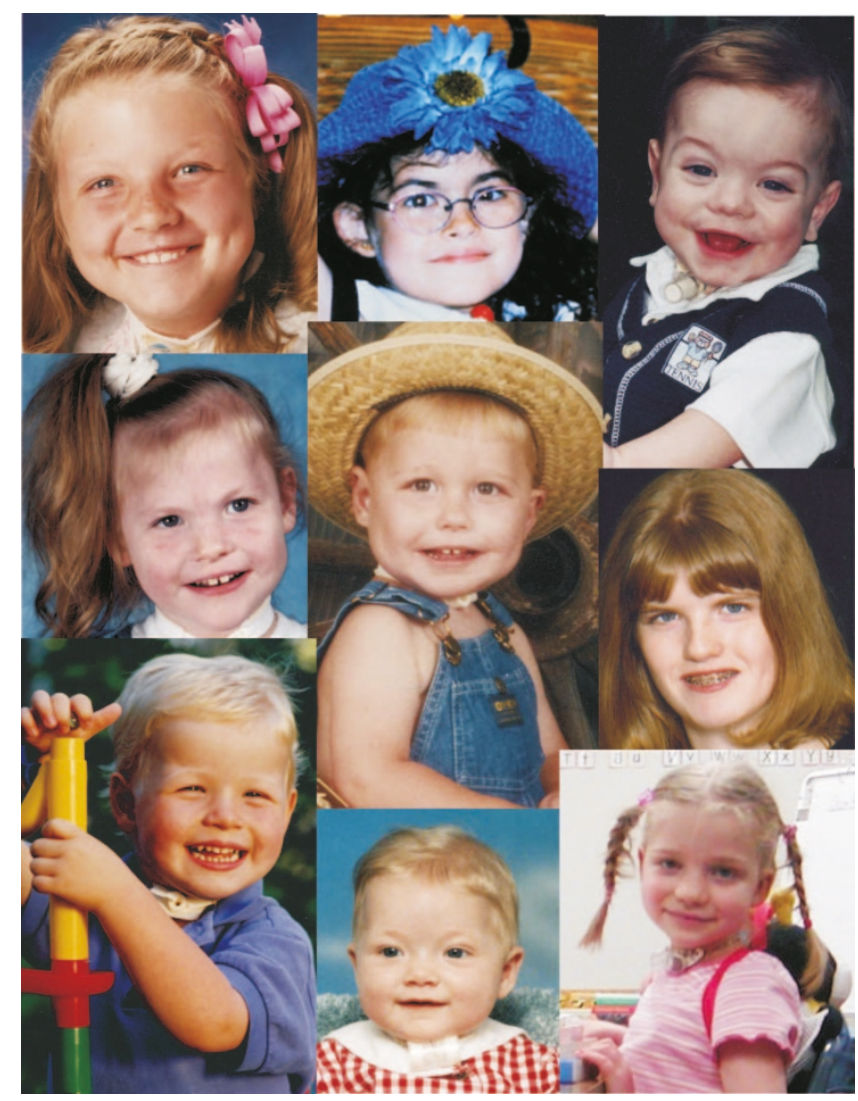

Figure 1. Composite of casual photographs from children with CCHS. These casual photographs of children with CCHS are examples of the photos that prompted the authors to consider a characteristic facies in CCHS.

develops into facial structures or viscerocranium $(12,13)$. Further, the original neuraxial order of the rhombencephalic neural crest is strictly maintained throughout development of the viscerocranium (14), such that skeletal muscle connective tissue arising from a specific neural crest rhombomere exclusively attaches to neural crest-derived skeletal structures from the same rhombomere, leading to strictly conserved musculoskeletal patterning (14). These observations led to the hypothesis that the CCHS facial appearance could be quantified, and is related to the presence of a mutation in the neural crestdefining $P H O X 2 B$ gene, with severity of the facial patterns proportional to the number of polyalanine repeats.

\section{METHODS}

CCHS cases. A sample of 45 children and young adults diagnosed with CCHS, with validation confirmed by a mutation in $P H O X 2 B$, were enrolled in the study. The diagnosis of CCHS was based on the accepted definition in the American Thoracic Society statement on the diagnosis and management of idiopathic CCHS (1). Among the CCHS cases, nine $(20 \%)$ were also diagnosed with Hirschsprung disease, and one of these nine subjects was diagnosed with neuroblastoma. Of the cases, 13 have used mask ventilation at some point in the management of their disease. The sex ratio of the study sample was 1:1, with 22 females and 23 males. Age range at enrollment was $1.5-24.6$ y with a mean age of 11.5 (SD 6.4) y (Fig. 3). Ethnicity was assigned based on self-report, with all subjects reporting themselves as Caucasian.

Controls. A control group of 45 children and young adults was recruited, matched for ethnicity, gender, and age ( $\pm 3 \mathrm{mo}$ ) to the CCHS cases. After informed consent was obtained, a three-generation family history was taken for each control to ensure that no family member had a diagnosis of sudden infant death syndrome, Hirschsprung disease, CCHS, apparent life threatening event, primary (nonacquired) disorder of ANS dysregulation, or tumor of
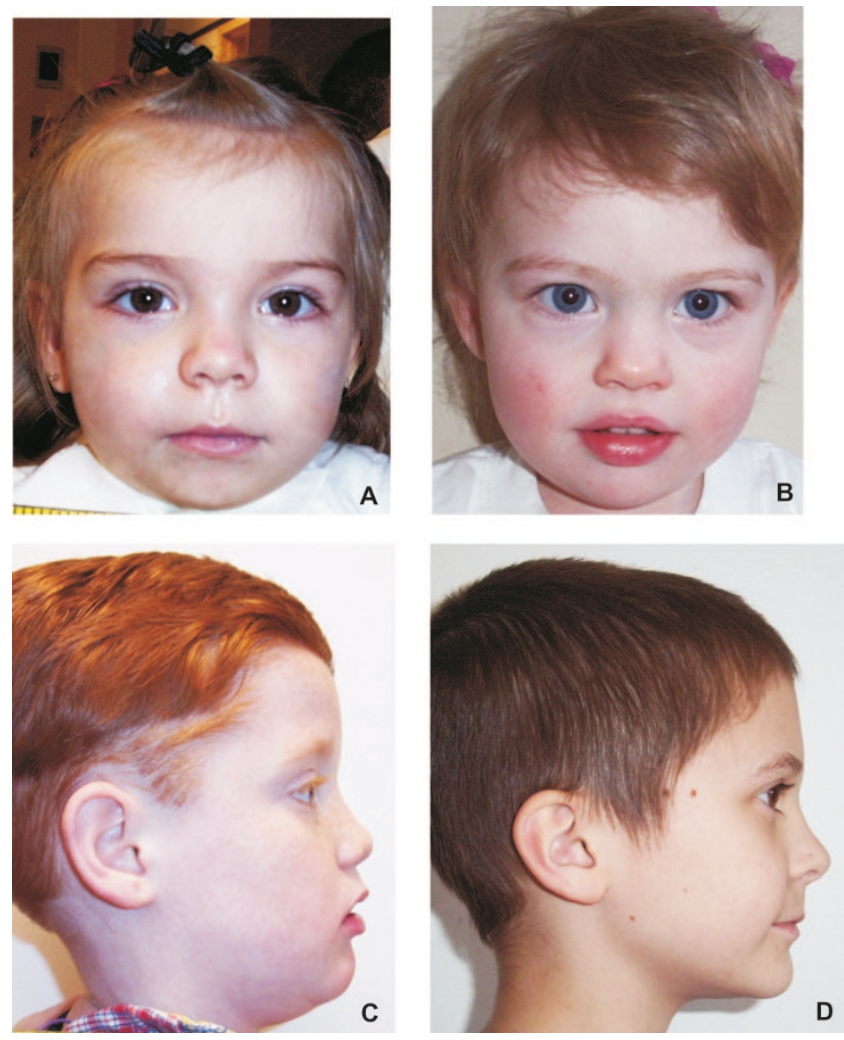

Figure 2. Photographs of representative CCHS and control subjects. These two children with CCHS both have the representative facies. The girl with CCHS (A) has a PHOX2B genotype of 20/25, and the boy with CCHS $(C)$ has a 20/27 genotype. Note the decreases in sloping of the forehead, upper face height, upper facial inclination, nasolabial angle, upper and lower lip heights, as well as the inferior inflection of the lateral vermillion border of the upper lip. Age-, gender-, and ethnicity-matched controls are also shown ( $B$ and $D$ ).
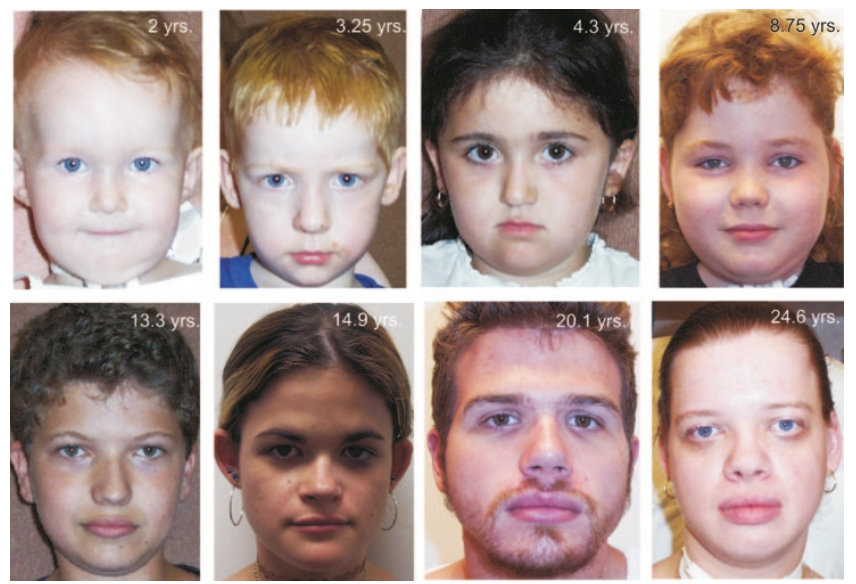

Figure 3. Characteristic facies in CCHS from ages 2.0 to $24.6 \mathrm{y}$ of age. These photographs of males and females with CCHS demonstrate the characteristic facies at a broad range of ages.

neural crest origin. Among the matched controls, the age range at enrollment was 1.5-24.7 y with a mean age of 10.9 (SD 6.1) y. Cases and controls did not differ statistically on age $(p>0.05)$.

Institutional review board approval. This study was approved by the Rush University Medical Center and the University of Pittsburgh institutional review boards. Informed consent and HIPAA authorization were obtained from all subjects and their parents or legal guardians.

DNA preparation. Blood (3-10 $\mathrm{mL}$ ) by venipuncture or a buccal swab was obtained and collected into an EDTA tube. Genomic DNA was isolated utilizing a Puregene reagent kit (Gentra, Minneapolis, MN) according to the 
manufacturer's instructions. DNA samples were saved in Tris-EDTA hydration buffer at $-80^{\circ} \mathrm{C}$ before genotyping.

Genotyping and sequence analysis of $\mathrm{PHOX} 2 \mathrm{~B}$ polyalanine repeat sequence. The $P H O X 2 B$ exon 3 region coding for the polyalanine repeat was amplified with primer pair 5'-CCAGGTCCCAATCCCAAC-3' (forward) and 5'-GAGCCCAGCCTTGTCCAG-3' (reverse) as described previously(2) (patent pending). For the case where the genotype was normal (20 polyalanine repeats on each allele) but the child had the CCHS/Hirschsprung disease/ neuroblastoma phenotype, $P H O X 2 B$ exons 1, 2, and 3 were amplified with primer pairs (15) and sequenced as described previously (2).

Photography protocol. Photographs of all study subjects were obtained using a Kodak EasyShare DX3700 or DX6340 digital camera (Rochester, $\mathrm{NY}$ ). The photographs were taken in a uniform manner in frontal view as well as both right and left profile views. A 12-inch ruler was held at the root of the neck of the subject in each picture for scale. A representative girl and boy with CCHS (and matched controls) are shown in Figure 2.

Photogrammetry technique. The technique of making measurements from photographs was validated by Farkas et al. (16), however, it was found that only 20 of the possible 104 measures were reliable when compared with standard anthropometry. This largely precluded the use of normative data derived from direct anthropometry for comparison purposes and necessitated that photogrammetric data be obtained from both cases and controls. Additionally, because Nechala et al. (17) demonstrated consistency among measures on digital pictures from a variety of cameras, we justified the use of digital photographs for the anthropometric measurements. All measurements were taken using CorelDraw 12 (Ottawa, ON, Canada), an updated version of the program used by Nechala et al. (17) for digital two-dimensional photogrammetry. Within the program, the digital images were rotated to place the subject in the Frankfurt horizontal position, the standard position for photo- grammetry. Also, each picture was scaled to full size so that measures taken on the photographs represented actual dimensions, with each measure taken to the hundredth of a millimeter. Seventeen linear and 6 angular measurements along with 9 indices computed as ratios of linear dimensions were obtained for each subject $(18,19)$. These measurements and indices are described in Table 1. Visual representation of the facial landmarks required to take these measurements is provided in Figure 4. In addition, the lateral segment of vermillion border on the upper lip was assessed for the presence of an inferior inflection. All measurements were made in a uniform manner, by one author (E.S.T.), who was blinded to the clinical diagnosis of CCHS as well as to the results of the genetic testing. Repeat measures were taken on all subjects, to assess intraobserver reliability.

Measurement error analysis. For the continuous variables, intraobserver precision was assessed by calculating intraclass correlation coefficients (20). For categorical variables, Kappa correlations were calculated.

Univariate statistics. For continuous variables, paired $t$ tests were used to test for differences between CCHS cases and their matched controls. A $\chi^{2}$ test was used to investigate whether inferior inflection of the lateral segment of vermillion border on the upper lip ("lip trait") was present more frequently in cases versus controls. All univariate tests were carried out both with the genders pooled and separated.

Multivariate statistics. Variables found to differ significantly $(p<0.05)$ from univariate tests were then entered in a stepwise logistic regression to identify the combination of variables that best discriminate between CCHS cases and controls.

Genotype/phenotype correlation. Two separate methods were used to investigate the relationship between genotype and phenotype. Within the CCHS sample, partial correlation coefficients were calculated between repeat number and anthropometric variables, adjusting for sex, age, Hirschsprung

Table 1. Photogrammetry linear and angular measurements and indices*

\begin{tabular}{|c|c|c|c|c|}
\hline Region & Measurement name & Plane of measure & View & Landmarks* \\
\hline \multicolumn{5}{|l|}{$\begin{array}{l}\text { Linear and angular } \\
\text { measurements }\end{array}$} \\
\hline \multirow[t]{2}{*}{ Head } & Supraorbital breadth & Linear horizontal & Frontal & $f z-f z$ \\
\hline & Forehead inclination & Inclination from vertical & Lateral & \\
\hline \multirow[t]{6}{*}{ Face } & Maximum face breadth & Linear horizontal & Frontal & zy-zy \\
\hline & Mandible breadth & Linear horizontal & Frontal & go-go \\
\hline & Lower face height & Linear vertical & Frontal & sn-gn \\
\hline & Mandible height & Linear vertical & Frontal & sto-gn \\
\hline & Upper face inclination & Inclination from vertical & Lateral & g-sn \\
\hline & Lower face inclination & Inclination from vertical & Lateral & sn-pg \\
\hline \multirow[t]{2}{*}{ Orbits } & Intercanthal width & Linear horizontal & Frontal & en-en \\
\hline & Biocular width & Linear horizontal & Frontal & ex-ex \\
\hline \multirow{2}{*}{ Nose } & Nasolabial angle & Angular & Lateral & \\
\hline & Nasal bridge inclination & Inclination from vertical & Lateral & \\
\hline \multirow[t]{3}{*}{ Orolabial Region } & Upper lip height & Linear vertical & Frontal & sn-sto \\
\hline & Lower lip height & Linear vertical & Frontal & sto-sl \\
\hline & Lateral lip height & Linear vertical & Frontal & sbal-ls' \\
\hline Ears & Ear length & Linear vertical & Lateral & sa-sba \\
\hline
\end{tabular}

Craniofacial indices

Head

Face

Orbits

Nose
Forehead-supraorbital index Forehead-face height index

Facial index

Mandible-face width

Upper facial index

Intercanthal index

Nasal index

Nose-face width

Nose-face height $\operatorname{tr}-\mathrm{g} / \mathrm{fz}-\mathrm{fz}$

tr-g/n-gn

n-gn/zy-zy

go-go/zy-zy

n-sto/zy-zy

en-en/ex-ex

al-al/n-sn

al-al/zy-zy

n-sn/n-gn
Measures relative forehead height

Measures relative forehead height

Measures relative face height

Measures relative mandible breadth

Measures relative upper face height

Measures relative intercanthal width

Measures relative nose width

Measures relative nose width

Measures relative nose length

* Refer to Figure 4 for photographs indicating facial landmarks used in taking measurements and deriving indices. 


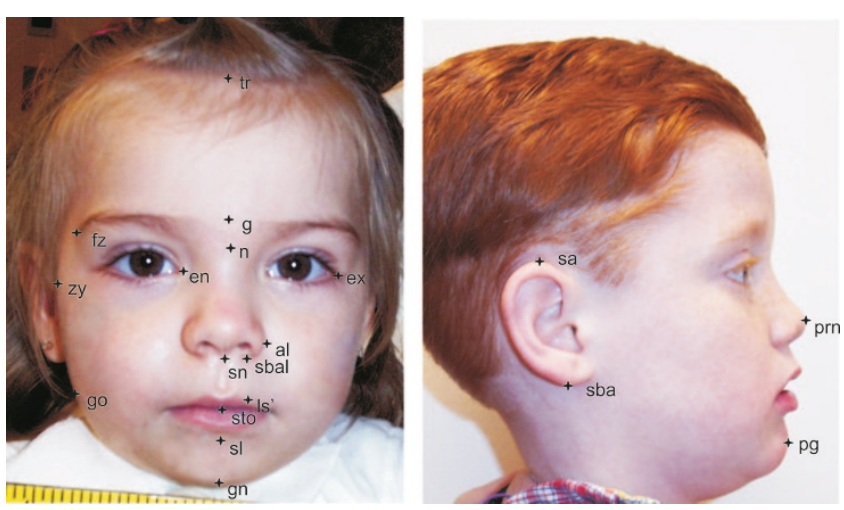

Figure 4. Identification of anthropometric landmarks to determine measurements used and described in Table 1. $t r$, trichion; $g$, glabella; $f z$, frontozygomaticus; $n$, nasion; $e x$, exocanthion; en, endocanthion; $z y$, zygion; sa, superaurale; $s b a$, subaurale; $a l$, alare; prn, pronasale; $s n$, subnasale; $s b a l$, subalare; $l s^{\prime}$, labiale superius'; sto, stomion; go, gonion; sl, sublabiale; gn, gnathion; $p g$, pogonion. As an example, upper face height is measured between the landmarks nasion, shown here as "n", and stomion, shown as "sto"; likewise, nasolabial angle is an angular measure between the columella of the nose and the upper lip with the axis at subnasale, shown as "sn".

disease, neural crest tumor, and use of facial mask ventilation. The use of mask ventilation was included because of concern for potential facial flattening in the event of prolonged use during critical periods of growth. For the second method, CCHS cases were lumped into two categories based on repeat number in the PHOX2B mutation: group 1 was comprised of cases with 25 or 26 repeats and group 2 had all cases with $\geq 27$ repeats. The rationale for this distinction was based on a recent abstract (21) studying the CCHS phenotype. A significant difference in longest $r-r$ interval by Holter recording among children with 26 repeats versus 27 repeats $(2.3 \pm 1 \mathrm{~s}$ and $3.8 \pm 1 \mathrm{~s}$, respectively, $p=0.001$ ) was identified. Conditional logistic regression was then carried out, adjusting for the same variables as in the partial correlation analysis.

All statistical analyses were carried out using SPSS v.11.5 (Chicago, IL).

\section{RESULTS}

CCHS cases. Among the CCHS cases, all were heterozygous for a mutation in $P H O X 2 B$. Forty-four individuals had a polyalanine expansion mutation and one had a unique missense point mutation near the exon $2 / 3$ splice site $(\mathrm{G} 422 \mathrm{~A}=$ arginine $141 \rightarrow$ glutamine), which resulted in impaired splicing of intron 2 (22). The mean number of extra polyalanine repeats on the affected allele was 27 (SD 2) for the total group, as well as the male and female subgroups. The distribution for the number of extra repeats was as follows: 11 with 25 repeats, 11 with 26 repeats, 15 with 27 repeats, one with 28 repeats, one with 29 repeats, and five individuals with 30-33 repeats.

Controls. The PHOX2B genotype for all controls was 20/ 20.

Measurement error. The mean intraclass correlation coefficient was 0.97 (95\% confidence interval: 0.96-0.98) for the continuous variables. A Kappa correlation of 1 was observed for the single categorical variable included in the study (lip trait), indicating perfect agreement. Based on these results, no variables had to be excluded from the present study.

Univariate analyses. Results for the linear and angular measurements and ratios are provided in Table 2. In the combined data set (genders pooled), 12 variables differed significantly between CCHS cases and controls (5 after $p$ value correction for multiple tests), with the strongest results involving lip measurements. When the genders were investigated separately, male CCHS cases differed to a greater extent compared with their matched controls than did females. In particular, male CCHS cases possessed significantly increased orbital measurements (versus controls), whereas these measures were not significant in female CCHS cases versus controls. Among the CCHS cases, the most consistent variables (significant in females and males separately and combined) included decreased upper face height (n-sto), increased nasal tip protrusion (sn-prn), decreased nasolabial angle, decreased upper lip height (sn-sto), decreased facial index (ngn/zy-zy), and decreased upper facial index (n-sto/zy-zy). Inferior inflection of the lateral segment of vermillion border on the upper lip was present in $53.3 \%$ of CCHS cases and $13.3 \%$ of controls $(p<0.001)$ for the total group, with $54.5 \%$ versus $18.2 \%$ among the females $(p=0.028)$ and $52.2 \%$ versus $8.7 \%$ among the males $(p=0.004)$.

Multivariate logistic regression. Thirteen variables were entered initially, chosen based on a $p$ value $<0.05$ from univariate tests. After five steps, the predictive ability of the model was no longer significantly improved. Variables included in the regression model and resultant statistics are provided in Table 3 . This analysis predicted correctly $85.7 \%$ of the CCHS cases and $82.2 \%$ of the controls. These analyses took into consideration the potential confounding variables of Hirschsprung disease, neuroblastoma, and use of mask ventilation.

Genotype/phenotype correlation. Evidence for a significant negative relationship between number of repeats and four anthropometric measures was observed: mandible breadth (go-go) $(r=-.38 ; p=0.015)$, nasolabial angle $(r=-.34 ; p$ $=0.035)$, lateral lip height (sbal-1s') $(r=-.33 ; p=0.036)$ and mandible-face width index (go-go/zy-zy) $(r=-.35 ; p=$ 0.029). Results of conditional logistic regression did not identify variables that were significantly predictive of genotype category.

Statistical significance. In the summary of results above we have applied a significance level $(\alpha)$ of 0.05 because this study seeks validation of anecdotal clinical impressions and we wished to capture the full range of case-control differences. If a conservative Bonferroni correction were applied to the significance level (i.e. using a threshold of either 0.002 or 0.001 for significance), fewer than one-third of all significant findings in Table 2 would remain significant. At a minimum, our results provide trends that require a larger number of subjects to substantiate, but note that these results in 45 case-control pairs are consistent with our clinical observation of more than 100 cases of CCHS.

\section{DISCUSSION}

These results represent the first study quantitating the characteristic facial phenotype in children and young adults with PHOX2B-confirmed CCHS. The faces of subjects with CCHS were generally shorter and flatter, [evidenced by significantly decreased upper face height (n-sto), increased nasal tip protrusion (sn-prn), decreased nasolabial angle, and decreased 


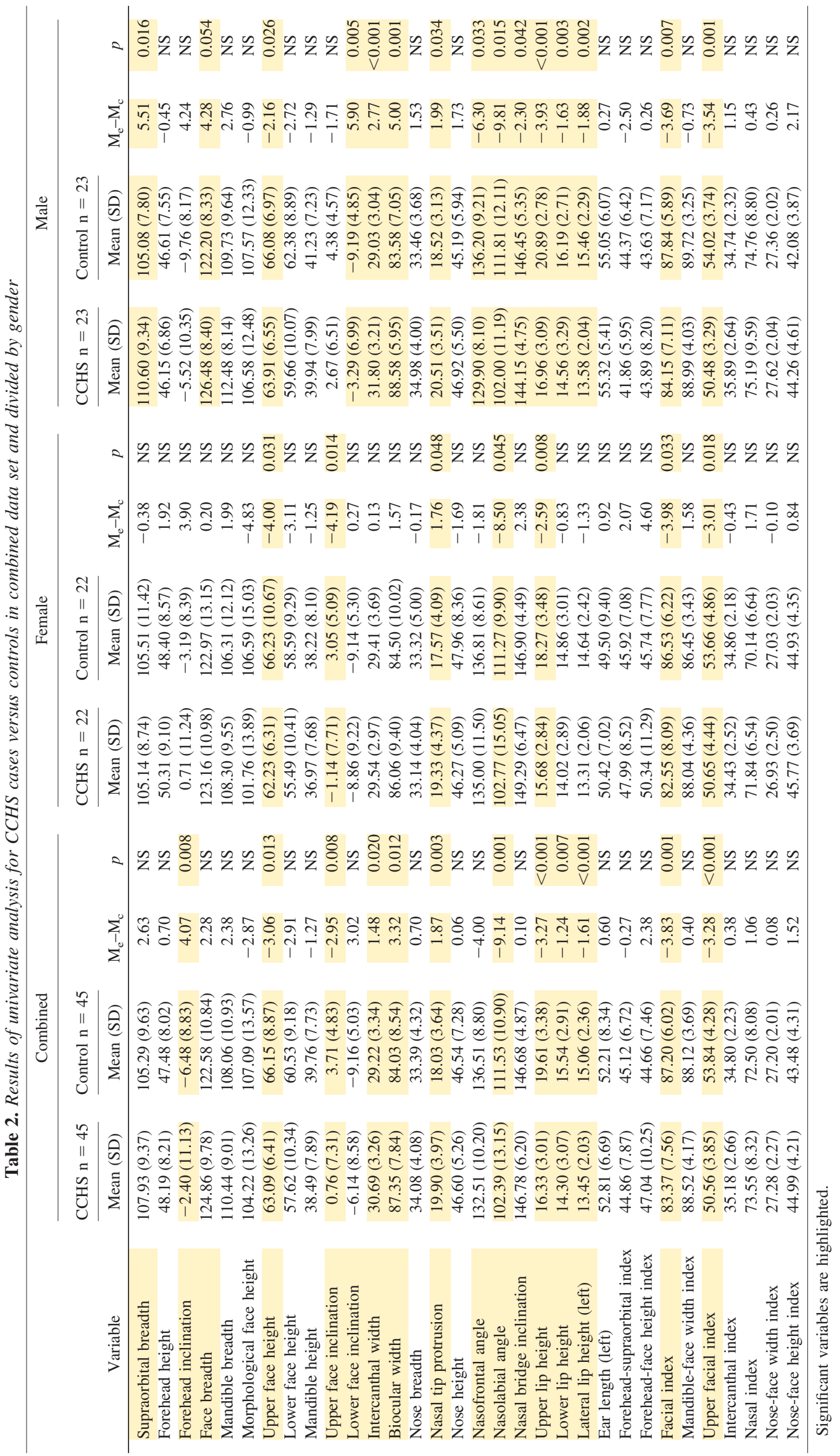


Table 3. Variables in multiple logistic regression: forward stepwise method $(n=45)$

\begin{tabular}{lcrrrc}
\hline \multicolumn{1}{c}{ Variable } & Entered at & \multicolumn{1}{c}{$B$} & Wald & $d f$ & $p$ \\
\hline Upper lip height & Step 1 & 1.997 & 6.964 & 1 & 0.008 \\
Biocular width & Step 2 & -2.207 & 8.979 & 1 & 0.003 \\
Upper facial height & Step 3 & -1.350 & 7.002 & 1 & 0.008 \\
Nasal tip protrusion & Step 4 & 1.367 & 7.451 & 1 & 0.006 \\
Lip trait* & Step 5 & 1.986 & 6.394 & 1 & 0.011 \\
Constant & & -1.452 & 4.955 & 1 & 0.026
\end{tabular}

$*$ Lip trait $=$ inferior inflection of the lateral segment of vermillion border of the upper lip.

upper lip height (sn-sto)] and typically showed an inferior inflection of the lateral segment of vermillion border on the upper lip. Furthermore, the significantly decreased facial index (n-gn/zy-zy) and decreased upper facial index (n-sto/zyzy) (such that the face is short relative to its width) results in the characteristic box-shaped face observed in CCHS. The results also suggest that male CCHS subjects are more strongly affected than females. Using five variables to characterize facies [upper lip height (sn-sto), biocular width (exex), upper facial height (n-sto), nasal tip protrusion (sn-prn) and the lip trait], $85.7 \%$ of the CCHS cases and $82.2 \%$ of the controls were correctly predicted. Evidence for a significant negative correlation between number of repeats and four anthropometric measures [measures get smaller for mandible breadth (go-go), nasolabial angle, lateral lip height (sbal-ls'), and mandible-face width index (go-go/zy-zy) as the number of polyalanine repeats increase] was observed. However, results of logistic regression indicate that no variables were predictive of genotype category.

Although data have been published indicating a relationship between the $\mathrm{PHOX} 2 \mathrm{~B}$ genotype and the CCHS phenotype as it relates to ANS dysregulation $(2,4,21)$ (increased number of polyalanine repeats associated with increased severity of ANS dysregulation), that relationship has not been explored for other aspects of the CCHS phenotype. The trend toward a correlation between increasing repeat number in $P H O X 2 B$ and a smaller angle or index among four facial measurements suggests a negative relationship may exist between the number of repeats and the extent of some aspects of the facial phenotype: as the number of repeats increases, the measurements seem to further decrease from normal. The limited number of cases with higher numbers of repeats (only five cases with 30-33 repeats) may have precluded us from finding a significant correlation between the number of polyalanine repeats and the severity of measures of the CCHS facial phenotype. Alternatively, the severity of the most extreme measures of the CCHS facial phenotype may not correlate with repeat size because any mutation that substantially disrupts the function of $P H O X 2 B$ results in these characteristic facial features - as suggested here by one child with a unique mutation in PHOX2B with the characteristic facies. This will need to be further clarified by similar studies of additional children with large expansion mutations and nonexpansion mutations in $P H O X 2 B$.
One previous publication (23) mentioned "facial dysmorphism" in an infant with CCHS and Hirschsprung disease. The dysmorphic features specifically summarized in that article are not among those that were significant in the current study; however, the photograph provided clearly shows the features that we have identified despite the infant's young age (death at $19 \mathrm{~d}$ ). Interestingly, three individuals have been reported with a deletion in 4p11-15 (24-26), in the region of the PHOX2B gene, but none of these cases are described as having alveolar hypoventilation. This would suggest that deletion of the PHOX2B gene does not result in the CCHS phenotype, thus supporting the concept of a dominant negative mechanism of action for $P H O X 2 B$ mutations in CCHS, rather than haploinsufficiency. Comparing the facial features among these three female cases with the case-control study presented here is less straightforward. A photograph was provided for only one of the case reports, but detailed description of the facial features was provided in all three publications. Specifically, the case with a translocation comparable to a 4p12-13 deletion and Hirschsprung disease was described as having microcephaly, frontal bossing, prominent forehead, very short nose with slightly anteverted nostrils, high nasal bridge, prominent philtral borders, and bilateral epicanthus; the case with the 4p1115.2 deletion was described as having microcephaly, a narrow face, short palpebral fissures, decreased outer canthal distance, ptosis, prominent nasal bridge, a long nose, and small triangular ears with attached lobes; the case with the 4p12-15 deletion was described as having the left half of skull flattened, epicanthic folds and antimongoloid slant of eyes, ears deformed with broad slightly irregular flattened helix and fixed lobes, prominent glabella, broad root of nose, asymmetry of shape of nose and localization of the nasal septum. The dissimilarity between these descriptions and those provided in our case-control study suggests that haploinsufficiency of PHOX2B may not result in sufficient reduction of function to generate the facial appearance of CCHS, supporting the dominant negative hypothesis. However, the patients with the large deletions described had many genes deleted and effects related to haploinsufficiency of other genes may have obscured the $P H O X 2 B$ effect on facial phenotype.

We identify two potential limitations of our study. First is the use of only one control per CCHS case. Ideally, three to five controls would be obtained and studied for each CCHS case to make broad generalizations about differences between individuals with CCHS and the general population. We feel strongly, however, that the statistical results of this present study support our clinical observations of the characteristic box-shaped face seen in individuals with CCHS. A second potential limitation is the inclusion of children with CCHS who ever used a mask for ventilation. Because so many children have been transitioned to noninvasive mask ventilation, an initial concern was that use of the face mask might confound our results. After controlling for use of mask ventilation as a possible confounder, we report no significant differences overall for tracheostomy versus mask including specifically those variables that might be impacted by mask ventilation: upper face inclination ( $\mathrm{g}$-sn), lower face inclination (sn-gn), nasal tip protrusion (sn-prn), nasofrontal angle, 
and nasal bridge inclination (the area encompassed by the mask). Thus, it seems that the use of mask ventilation does not obscure the intrinsic differences in facial measures comparing cases with CCHS to matched controls at the point in time captured by the photographs used in this study. The possibility remains that there may be key stages of facial development that could be compromised by the use of mask ventilation. This would need to be assessed with a longitudinal study following the same individuals from infancy to adulthood, which was not explored in this study.

The current study was undertaken primarily in children (mean age, $11.5 \mathrm{y}$; SD, 6.4), but the facial phenotype may also be useful for characterization of older children and adults with unexplained hypercarbia. For example, we recently identified an adult in whom CCHS was not suspected until age 35 when he presented with a carbon dioxide value in excess of $120 \mathrm{~mm}$ $\mathrm{Hg}$ after surgical intervention for obstructive sleep apnea (27). He was found to have a $P H O X 2 B$ mutation (genotype 20/25), and has features of the characteristic facial phenotype described here. Subsequent to that report, we determined that another young man who presented at age 23 y (28), who is now 35 y old, shares the same PHOX2B genotype of 20/25 and likewise has many features of the characteristic CCHS facies (Antic N, personal communication).

This study has validated anecdotal observations about facial appearance in children with CCHS and has the potential to be a diagnostic tool in the categorization of individuals with CCHS and related disorders. A much larger study of faces from a variety of individuals with CCHS and similar disorders will help elucidate these relationships and ascertain the effects of genes involved in the embryologic origin of the ANS on facial structure. As such, the collaboration between clinical and basic science investigators and the centralization of care of children and adults with rare disease have the potential for elucidating specific phenotypic components in seemingly complex diseases.

Acknowledgments. The authors thank the children and young adults with CCHS, as well as the control subjects who graciously shared their photographs and their DNA. We also thank William B. Dobyns, M.D., Ph.D., for his insightful observations in this project.

\section{REFERENCES}

1. Weese-Mayer DE, Shannon DC, Keens TG, Silvestri JM 1999 American Thoracic Society Statement. Idiopathic congenital central hypoventilation syndrome: diagnosis and management. Am J Respir Crit Care Med 160:368-373

2. Weese-Mayer DE, Berry-Kravis EM, Zhou L, Maher BS, Silvestri JM, Curran ME, Marazita ML 2003 Idiopathic congenital central hypoventilation syndrome: analysis of genes pertinent to early autonomic nervous system embryologic development and identification of mutations in PHOX2b. Am J Med Genet 123A:267-278
3. Amiel J, Laudier B, Attié-Bitach T, Trang H, de Pontual L, Gener B, Trochet D, Etchevers H, Ray P, Simonneau M, Vekemans M, Munnich A, Gaultier C, Lyonnet S 2003 Polyalanine expansion and frameshift mutations of the paired-like homeobox gene $P H O X 2 B$ in congenital central hypoventilation syndrome. Nat Genet 33:459461

4. Matera I, Bachetti T, Puppo F, Di Duca M, Morandi F, Casiraghi GM, Cilio MR, Hennekam R, Hofstra R, Schöber JG, Ravazzolo R, Ottonello G, Ceccherini I 2004 $P H O X 2 B$ mutations and polyalanine expansions correlate with the severity of the respiratory phenotype and associated symptoms in both congenital and late onset central hypoventilation syndrome. J Med Genet 41:373-380

5. Sasaki A, Kanai M, Kijima K, Akaba K, Hashimoto M, Hasegawa H, Otaki S, Koizumi T, Kusuda S, Ogawa Y, Tuchiya K, Yamamoto W, Nakamura T, Hayasaka K 2003 Molecular analysis of congenital central hypoventilation syndrome. Hum Genet 114:22-26

6. Weese-Mayer DE, Berry-Kravis EM 2004 Genetics of congenital central hypoventilation syndrome: lessons from a seemingly orphan disease. Am J Respir Crit Care Med 170:16-21

7. Trochet D, O'Brien LM, Gozal D, Trang H, Nordenskjöld A, Laudier B, Svensson PJ, Uhrig S, Cole T, Munnich A, Gaultier C, Lyonnet S, Ameil J 2005 PHOX2B genotype allows for prediction of tumor risk in congenital central hypoventilation syndrome. Am J Hum Genet 76:421-426

8. Dubreuil V, Hirsch MR, Jouve C, Brunet JF, Goridis C 2002 The role of PHOX2b in synchronizing pan-neuronal and type-specific aspects of neurogenesis. Development 129:5241-5253

9. Lo L, Morin X, Brunet JF, Anderson DJ 1999 Specification of neurotransmitter identity by Phox 2 proteins in neural crest stem cells. Neuron 22:693-705

10. Pattyn A, Morin X, Cremer H, Goridis C, Brunet JF 1999 The homeobox gene Phox $2 b$ is essential for the development of autonomic neural crest derivatives. Nature 399:366-370

11. Pattyn A, Morin X, Cremer H, Goridis C, Brunet JF 1997 Expression and interactions of the two closely related homeobox genes Phox $2 a$ and Phox $2 b$ during neurogenesis. Development 124:4065-4075

12. Le Douarin NM, Ziller C, Couly GF 1993 Patterning of neural crest derivatives in the avian embryo: in Vivo and in Vitro studies. Dev Biol 159:24-49

13. Couly GF, Coltey PM, Le Douarin NM 1993 The triple origin of skull in higher vertebrates: a study in quail-chick chimeras. Development 117:409-429

14. Köntges G, Lumsden A 1996 Rhombencephalic neural crest segmentation is preserved throughout craniofacial ontogeny. Development 122:3229-3242

15. Garcia-Barceló M, Sham MH, Lui VC, Chen BL, Ott J, Tam PK 2003 Association study of $\mathrm{PHOX} 2 \mathrm{~B}$ as a candidate gene for Hirschsprung's disease. Gut 52:563-567

16. Farkas LG, Bryson W, Klotz J 1980 Is photogrammetry of the face reliable? Plast Reconstr Surg 66:346-355

17. Nechala P, Mahoney J, Farkas LG 1999 Digital two-dimensional photogrammetry: a comparison of three techniques of obtaining digital photographs. Plast Reconstr Surg 103:1819-1825

18. Farkas LG 1994 Anthropometry of the Head and Face, 2nd ed. Raven Press, New York, pp 21-51

19. Farkas LG, Munro IR 1987 Anthropometric Facial Proportions in Medicine, p 344 Charles C. Thomas, Springfield, IL

20. Fleiss JL 1986 The Design and Analysis of Clinical Experiments, p 432, John Wiley and Sons, New York

21. Silvestri JM, Weese-Mayer DE, Berry-Kravis EM 2004 Congenital central hypoventilation syndrome: $\mathrm{PHOX} 2 b$ genotype and sinus pause phenotype. Am J Respir Crit Care Med 169:A263

22. Berry-Kravis EM, Zhou L, Rand C, Weese-Mayer DE 2005 Unique mutations in children with congenital central hypoventilation syndrome (CCHS). PAS Rep $57: 2289$

23. Minutillo C, Pemberton PJ, Goldblatt J 1989 Hirschsprung's disease and Ondine's curse: further evidence for a distinct syndrome. Clin Genet 36:200-203

24. Benailly HK, Lapierre JM, Laudier B, Amiel J, Attié T, De Blois MC, Vekemans M, Romana SP 2003 PMX2B, a new candidate gene for Hirschsprung's disease. Clin Genet 64:204-209

25. Francke U, Arias DE, Nyhan WL 1977 Proximal 4p-deletion: phenotype differs from classical 4p-syndrome. J Pediatr 90:250-252

26. Nielsen J, Fischer O, Rasmussen K, Sillesen I, Bernsen A, Saldana-Garcia P 1977 A 13-year-old girl with Wolf's syndrome and karyotype 46,XX, del(4) (pterp integral $15:: \mathrm{p} 12$ integral qter), $9 \mathrm{qh}+$. Increased risk of structural chromosome abnormalities in the progeny of mothers with 9qh+. J Ment Defic Res 21:119-126

27. Weese-Mayer DE, Berry-Kravis EM, Zhou L 2005 Adult identified with congenital central hypoventilation syndrome -mutation in $\mathrm{PHOX} 2 b$ gene and late-onset CHS. Am J Respir Crit Care Med 171:88

28. Antic N, McEvoy RD 2002 Primary alveolar hypoventilation and response to the respiratory stimulant almitrine. Intern Med J 32:622-624 